\title{
An Open Study of Sulforaphane-rich Broccoli Sprout Extract in Patients with Schizophrenia
}

\author{
Akihiro Shiina ${ }^{1,2}$, Nobuhisa Kanahara ${ }^{3}$, Tsuyoshi Sasaki ${ }^{1}$, Yasunori Oda ${ }^{4}$, Tasuku Hashimoto ${ }^{4}$, Tadashi \\ Hasegawa $^{5}$, Taisuke Yoshida ${ }^{6}$, Masaomi Iyo ${ }^{1,4,5,7}$, Kenji Hashimoto ${ }^{8}$ \\ ${ }^{1}$ Department of Child Psychiatry, Chiba University Hospital, ${ }^{2}$ Division of Law and Psychiatry, Chiba University Center for Forensic Mental \\ Health, ${ }^{3}$ Division of Medical Treatment and Rehabilitation, Chiba University Center for Forensic Mental Health, ${ }^{4}$ Department of Psychiatry, \\ Graduate School of Medicine, Chiba University, ${ }^{5}$ Department of Psychiatry, Chiba University Hospital, ${ }^{6}$ Kisarazu Hospital, ${ }^{7}$ Chiba University \\ Center for Forensic Mental Health, ${ }^{8}$ Division of Clinical Neuroscience, Chiba University Center for Forensic Mental Health, Chiba, Japan
}

\begin{abstract}
Objective: Schizophrenia is a mental disorder characterized by severe cognitive impairment. Accumulating evidence suggests a role for oxidative stress in the pathophysiology of schizophrenia. Sulforaphane (SFN) extracted from broccoli sprout is an agent with potent anti-oxidant and anti-inflammatory activity. In this study, we attempted to evaluate the effect of SFN on cognitive impairment in medicated patients with schizophrenia.

Methods: We recruited a total of 10 outpatients with schizophrenia, all of whom gave informed consent. Participants took 3 tablets of SFN, consisting of $30 \mathrm{mg}$ of SFN-glucosinolate per day, for 8 weeks. Clinical symptoms using the Positive and Negative Syndrome Scale (PANSS) and cognitive function using the Japanese version of CogState battery were evaluated at the beginning of the study and at week 8 .

Results: A total of 7 patients completed the trial. The mean score in the Accuracy component of the One Card Learning Task increased significantly after the trial. However, we detected no other significant changes in participants.

Conclusion: This result suggests that SFN has the potential to improve cognitive function in patients with schizophrenia
\end{abstract}

KEY WORDS: Schizophrenia; Sulforafan; Executive function; Clinical trial; CogState.

\section{INTRODUCTION}

Schizophrenia is a mental disorder characterized by severe cognitive impairment, as well as positive and negative symptoms. ${ }^{1)}$ Cognitive deficits in schizophrenic patients are thought to be related to impaired dorsolateral prefrontal cortex function and, its interactions with other brain regions, such as the parietal cortex, thalamus and striatum, as well as the influence of neurotransmitter systems, such as dopamine, $\gamma$-aminobutyric acid (GABA) and glutamate. ${ }^{2)}$ It can also be related to poor compliance with antipsychotic medication, a known risk factor for relapse and re-hospitalization. ${ }^{3,4)}$ Importantly, cognitive impairment is highly relevant to the functional outcome of schizophrenic patients, ${ }^{5)}$ making recovery from this state

\footnotetext{
Received: June 23, 2014 / Revised: September 30, 2014

Accepted: October 2, 2014

Address for correspondence: Akihiro Shiina, MD, PhD

Department of Child Psychiatry, Chiba University Hospital, 1-8-1

Inohana, Chuoh-ku, Chiba 260-8670, Japan

Tel: +81-43-222-7171, Fax: +81-43-226-2297

E-mail: shiina-akihiro@faculty.chiba-u.jp
}

vital for improving the quality of life for sufferers.

There have been numerous attempts to treat cognitive deficits in patients with schizophrenia. ${ }^{6)}$ Atypical antipsychotics improve several domains of cognitive function, especially working memory, executive function and attention, in schizophrenic patients. ${ }^{7-9)}$ Adjunctive regimens with agents such as alpha-7 nicotinic acetylcholine receptor agonists, ${ }^{10,11)}$ sigma-1 receptor agonists, ${ }^{12,13)}$ selective estrogen receptor modulators, ${ }^{14)}$ and D-amino acid oxidase inhibitors ${ }^{15)}$ have been examined in clinical trials. However, no gold standard has been established, partly due to the lack of adequate efficacy for any of the tested agents. $^{16)}$

Accumulating evidence suggest a role for oxidative stress in the pathophysiology of schizophrenia. ${ }^{17-19)}$ Oxidative damage may precipitate a range of cognitive deficits in patients with schizophrenia, ${ }^{20,21)}$ through decreased activity of plasma manganese superoxide dismutase ${ }^{22,23)}$ and/or mitochondrial function. ${ }^{24)}$ Based on this evidence, several studies evaluating the effect of antioxidants on schizophrenia have been conducted. ${ }^{25)}$ Recent studies report

(c) This is an Open-Access article distributed under the terms of the Creative Commons Attribution Non-Commercial License (http://creativecommons.org/licenses/by-nc/3.0) which permits unrestricted non-commercial use, distribution, and reproduction in any medium, provided the original work is properly cited. 
on the possible positive effects of antioxidant treatments, such as $N$-acetyl cysteine, ${ }^{26)}$ vitamins, ${ }^{27)}$ extract of Ginkgo biloba $^{28)}$ and essential polyunsaturated fatty acids. ${ }^{29-32)}$ However, to our knowledge there have been few reports of antioxidants improving cognitive deficits in schizophrenic patients. No alleviation of cognitive deficits was found in a placebo-controlled trial with omega 3 fatty acids, ${ }^{33)}$ whereas L-carnosine adjunctive therapy showed beneficial effects on some domains of cognitive function along with considerable adverse effects, in a placebo-controlled trial. ${ }^{34)}$

Sulforaphane (SFN) is a molecule belonging to the isothiocyanate group of organosulfur compounds found in broccoli sprouts. It is known to have potent anti-oxidant and anti-inflammatory activity. ${ }^{35)}$ In clinical settings, SFN has been proposed as an alternative treatment against gastric tumors and other physical diseases. ${ }^{36)}$ Previously, we reported that SFN attenuated behavioral abnormalities in mice after administration of methamphetamine ${ }^{37)}$ or phencyclidine, ${ }^{38)}$ suggestive of a potential therapeutic potency in schizophrenia. Recently, we found that SFN improved cognitive deficits in phencyclidine-treated mice (Shirai et $a l$. , in preparation). To date, there are no published reports examining the beneficial effects of SFN in schizophrenia. To evaluate the efficacy of SFN on cognitive function, we conducted an open trial of SFN augmentation, in stable patients with schizophrenia. In addition, we measured serum levels of brain-derived neurotrophic factor (BDNF) in patients at baseline and 8-weeks, since it was reported that BDNF shows neurotrophic activity against oxidative stress. $^{39,40)}$

\section{METHODS}

\section{Study Design and Subjects}

This is an open-label, preliminary clinical trial for patients with schizophrenia. The recruited outpatients from Chiba University Hospital were aged between 20 and 65 years of age, with a diagnosis of schizophrenia meeting criteria defined by the Diagnostic and Statistical Manual of Mental Disorders 4th edition text revised (DSM-IV-TR), ${ }^{41)}$ were being prescribed a single atypical antipsychotic drug (aripiprazole, blonanserin, olanzapine, paliperidone, perospirone, quetiapine, or risperidone) at a fixed dose, for at least 4 weeks before study entry. Both sexes were recruited; however, pregnant women and new mothers were excluded. Patients with intellectual disabilities, developmental disorders, attention-deficit hyperactivity disorders, delirium, dementia or other types of cognitive im- pairment, substance misuse disorders (except caffeine or nicotine), eating disorders, or personality disorders were excluded. Patients who had previously been administered SFN over 8 weeks were also excluded.

\section{Measurement of Clinical Symptoms}

After giving written informed consent, participants received 3 tablets of SFN prepared by Kagome Co., Ltd. (Nagoya, Japan), totaling $30 \mathrm{mg}$ of SFN-glucosinolate per day, for 8 weeks. It is known that SFN-glucosinolate is metabolized to SFN in the body. We gave the each participant a recording sheet for them to confirm daily taking of SFN. We checked the sheet to evaluate their compliance in every visit.

We assessed each patient's medical condition every 2 weeks, using clinical interviews. Additionally, we performed blood tests at baseline, week 4, and week 8, to ensure the safety of SFN. Cognitive function was measured using the Japanese version of CogState (CogState Ltd., Connecticut, USA; http://cogstate.com) battery, which consists of a series of computed non-verbal tests. ${ }^{42)}$ In CogState, we chose the 5 subtests: Detection, Identification, One Card Learning, One Back Memory, and Two Back Memory. In Detection and Identification tasks, the primary performance measure was reaction time in milliseconds (speed), which was normalized using a $\log 10$ transformation. In the other tasks, the primary performance measure was the proportion of correct answers (accuracy), which was normalized using an arcsine square-root. $^{43)}$

In this trial, tests were performed at baseline and again at week 8. We also evaluated the psychiatric symptoms of participants using the Positive and Negative Syndrome Scale (PANSS), ${ }^{44)}$ and Clinical Global Impression (CGI) ${ }^{45)}$ at baseline, week 4 and week 8 . In addition, we measured the serum levels of BDNF in each participant at baseline and week 8 .

\section{Measurement of BDNF Serum Levels}

Serum samples from all subjects were collected between 9:00 and 15:00, and stored at $-80^{\circ} \mathrm{C}$ until use. Serum levels of BDNF were measured using the human BDNF ELISA kit (Aviscera Bioscience, Santa Clara, CA, USA). To minimize assay variance, serum levels of BDNF from each subject were measured on the same day. All experiments were performed in duplicate. Protocols were performed according to the manufacturer's instructions. The optical density of each well was measured using an automated microplate reader (Emax; Molecular 
Devices, Sunnyvale, CA, USA).

\section{Statistical Analysis}

On completion of clinical assessments, we evaluated data using IBM SPSS Statistics ver. 22 (Essentials for R; IBM Co., Armonk, NY, USA), based on intention-to-treat analysis, with the adapting last observation carried forward concept for unmeasured scores in participants who dropped out of the study. Considering the small sample size, we adopted Wilcoxon signed-rank test for statistical analyses. Values of $p<0.05$ (two-tailed) were considered

Table 1. Baseline demographics and clinical characteristics of participants

\begin{tabular}{lc}
\multicolumn{1}{c}{ Variable } & Data \\
\hline Age (yr) & $42.7 \pm 11.0$ \\
Gender (male : female) & $4: 6$ \\
Schizophrenia subtype & 2 \\
Disorganized & 1 \\
Catatonic & 7 \\
Paranoid & $18.9 \pm 10.0$ \\
Duration of illness (yr) & \\
CGl-S & 1 \\
2 (borderline) & 2 \\
3 (mildly ill) & 1 \\
4 (moderately ill) & 5 \\
5 (markedly ill) & 1 \\
7 (among the most extremely ill) & \\
Chief medication (mg/day) & 3 \\
Aripiprazole (15, 18, and 30) & 1 \\
Blonanserin (24) & 1 \\
Olanzapine (20) & 2 \\
Paliperidone (6 and 9) & 1 \\
Quetiapine (600) & 2 \\
Risperidone (2 and 3) &
\end{tabular}

Values are presented as mean \pm standard deviation or number only.

CGI-S, Clinical Global Impression - Severity of Illness. statistically significant in these analyses.

\section{RESULTS}

We recruited a total of 11 outpatients, who were given atypical antipsychotic mono-therapy against schizophrenia. This trial ran from October 2012 to July 2013. Of the 11 participants, one withdrew consent before initial assessments citing personal reasons, leaving a total of 10 patients. The baseline demographics, and clinical and treatment characteristics of patients are shown in Table 1. Three participants dropped out during the trial, whose scores of CGI-S were 5, 5, and 7. The reasons were: poor compliance with regular antipsychotic medication, withdrawal of consent to participate and the need to deal with other physical symptoms. No withdrawals were proven to be due to SFN related adverse events. Consequently, a total of 7 participants completed the trial. All completers took SFN with good adherence (over $90 \%$ ).

The mean scores in the Accuracy component of the One Card Learning Task (OCLT) showed a significant increase from 0.88 to 0.95 (Wilcoxon signed rank test: $p=0.043$ ). The other scores in CogState battery did not change significantly. The mean PANSS total scores $(91.7 \pm 16.2$ at baseline, 91.6 \pm 16.6 after 4 weeks and $91.0 \pm 16.3$ after 8 weeks; mean \pm standard deviation) showed no changes either. We also compared serum levels of BDNF after taking SFN for 8 weeks to baseline data in the completers, however no statistical differences were detected (Table 2).

\section{DISCUSSION}

These results highlight the possible use of SFN as an adjunctive therapy to improve cognitive function in patients

Table 2. Change of symptoms, brain-derived neurotrophic factor (BDNF) data, and cognitive function

\begin{tabular}{lcc}
\hline & Pre-medication & 8 Weeks \\
\hline PANSS (n=10, LOCF) & & $20.3 \pm 4.2$ \\
Positive scale & $20.5 \pm 3.8$ & $24.0 \pm 8.5$ \\
Negative scale & $25.2 \pm 8.6$ & $46.7 \pm 6.9$ \\
General psychopathology scale & $46.0 \pm 7.1$ & $91.0 \pm 16.3$ \\
Total score & $91.7 \pm 16.2$ & $23.9 \pm 9.26$ \\
Serum BDNF (ng/mL) & $31.5 \pm 2.89$ & 0.105 \\
CogState (n=10, LOCF) & & 0.236 \\
Detection (Speed) & $2.66 \pm 0.16$ & $2.68 \pm 0.15$ \\
Identification (Speed) & $2.83 \pm 0.12$ & $2.84 \pm 0.13$ \\
One Card Learning (Accuracy) & $0.88 \pm 0.083$ & $0.96 \pm 0.15$ \\
One Back Memory (Accuracy) & $1.20 \pm 0.23$ & $1.21 \pm 0.22$ \\
Two Back Memory (Accuracy) & $1.08 \pm 0.26$ & 0.149 \\
\hline
\end{tabular}

Values are presented as mean \pm standard deviation.

PANSS, Positive and Negative Syndrome Scale; LOCF, last observation carried forward. ${ }^{*} p<0.05$ (Wilcoxon signed-rank test). 
with schizophrenia. To the best of our knowledge, this is the first report highlighting an effect for SFN augmentation in medicated patients with schizophrenia.

The CogState battery is a valid measurement of cognitive function, similar to the MATRICS (Measurement and Treatment Research to Improve Cognition in Schizophrenia; http://www.matricsinc.org), ${ }^{46,47)}$ which is authorized as a standard evaluative tool for the components of cognitive impairment in patients with schizophrenia ${ }^{48)}$ and other diseases. ${ }^{49,50)}$ Its test-retest reliability is high enough for use in clinical evaluations, ${ }^{43)}$ while it was also assessed to have intermediate reliability and validity in a systematic review. ${ }^{51)}$ It is performed in Japanese patient cohorts with high validity. ${ }^{42)}$

The OCLT is a continuous visual recognition learning task that primarily estimates visual recognition learning, attention and short-term memory. ${ }^{52-54)}$ Its accuracy score has negative association with scores from the Mini Mental Examination in healthy controls, which indicates it should be able to detect cognitive impairment with a considerable degree of sensitivity. ${ }^{43)}$ In this study, we found that SFN improved mean scores on the OCLT in patients with schizophrenia, implying that it may have the potential to enhance domains of cognitive function, such as attention-focusing.

On the other hand, other scores than OCLT did not change in this study. One Back Memory Task (OBMT) and Two Back Memory Task (TBMT) also reflect working memory and attention of examinees. In this study, the baseline accuracy rates of the participants were high enough ( $93 \%$ in OBMT and $88 \%$ in TBMT). Therefore, it was possible that the improvement of cognitive function could not be detected with these tasks because of ceiling effect.

SFN is also reported to attenuate oxidative stress caused by antipsychotics. ${ }^{55)}$ It is likely that SFN could offer safer and beneficial therapy, avoiding the chronic problems frequently induced by antipsychotic medication, in addition to improving cognitive function.

There currently lack of consensus around practice effects in the CogState battery. In a few studies using healthy volunteers and schizophrenic patients, no practice effects were observed at the one-month test-retest interval, in some measures of CogState battery. ${ }^{56,57)}$ In studies of patients with dementia, CogState showed acceptable stability, with no or minimal practice effects, even at short test-retest intervals. ${ }^{58,59)}$ On the other hand, a study in which some computerized neurocognitive assessment tools were adapted to an active duty military sample, there was a finding that the intraclass correlation score of the OCLT was not high, compared with other tasks in CogState. ${ }^{60)}$ An open label, multinational methodology study, reported that the composite score on CogState battery showed a moderate practice effect on the second administration compared to the first administration (effect size $=0.28$, $95 \%$ confidence interval $[\mathrm{CI}]-0.05$ to 0.61 ); however, no further practice effect was observed between the second and the third administrations (effect size $=-0.05$, $95 \%$ CI -0.03 to 0.46 ), suggesting a saturation of practice effects at the second administration. ${ }^{61)}$ In contrast, another study in which repetitive examinations were performed over 12 months in elderly healthy people, there was a small improvement in group performance from baseline to the 12-month visit in the accuracy of performance in the OCLT, but the study failed to identify practice effects between the first two visits over a three month period. ${ }^{52)}$ To sum up, although practice effects in CogState cannot be ignored, it is highly unlikely to be a confounding factor in the results presented in this study.

Multiple data implicates BDNF in the pathophysiology of schizophrenia. ${ }^{62,63)}$ Some papers have specified a role for BDNF in cognitive deficits associated with schizophrenia. ${ }^{64,65)}$ Although we detected a recovery of some cognitive deficits, we found no change of serum BDNF in patients after eight weeks of SFN supplementation. A further study on the role of BDNF in the therapeutic mechanisms of SFN will be needed.

In summary, our clinical trial indicates that SFN has the potential to improve some domains of cognitive function in patients with schizophrenia. Further research is required including, randomized, double-blind, placebo-controlled trials, along with the measurement of potential biological markers for oxidative stress. Larger trials conducted over longer periods will be necessary to determine the efficacy and safety of SFN use in schizophrenia.

In conclusion, our pilot study suggests that supplementation therapy of SFN-rich broccoli sprout extract may have the potential to improve cognitive deficits in patients with schizophrenia.

\section{Acknowledgments}

We are greatly thankful to Kazushi Tsuru for measuring cognitive function of the participants using CogState during this trial.

\section{REFERENCES}

1. Elvevåg $\mathrm{B}$, Goldberg TE. Cognitive impairment in schizophrenia is the core of the disorder. Crit Rev Neurobiol 2000;14:1-21. 
2. Barch DM, Ceaser A. Cognition in schizophrenia: core psychological and neural mechanisms. Trends Cogn Sci 2012; 16:27-34.

3. Llorca PM. Partial compliance in schizophrenia and the impact on patient outcomes. Psychiatry Res 2008;161:235-247.

4. Robinson DG, Woerner MG, Alvir JM, Bilder RM, Hinrichsen GA, Lieberman JA. Predictors of medication discontinuation by patients with first-episode schizophrenia and schizoaffective disorder. Schizophr Res 2002;57:209-219.

5. Green MF. Cognitive impairment and functional outcome in schizophrenia and bipolar disorder. J Clin Psychiatry 2006;67 Suppl 9:3-8.

6. Ibrahim HM, Tamminga CA. Treating impaired cognition in schizophrenia. Curr Pharm Biotechnol 2012;13:1587-1594.

7. Harvey PD, Keefe RS. Studies of cognitive change in patients with schizophrenia following novel antipsychotic treatment. Am J Psychiatry 2001;158:176-184.

8. Meltzer HY, McGurk SR. The effects of clozapine, risperidone, and olanzapine on cognitive function in schizophrenia. Schizophr Bull 1999;25:233-255.

9. Sumiyoshi T, Higuchi Y, Uehara T. Neural basis for the ability of atypical antipsychotic drugs to improve cognition in schizophrenia. Front Behav Neurosci 2013;7:140.

10. Lieberman JA, Dunbar G, Segreti AC, Girgis RR, Seoane $\mathrm{F}$, Beaver JS, et al. A randomized exploratory trial of an a-7 nicotinic receptor agonist (TC-5619) for cognitive enhancement in schizophrenia. Neuropsychopharmacology 2013:38:968-975.

11. Shiina A, Shirayama Y, Niitsu T, Hashimoto T, Yoshida T, Hasegawa $\mathrm{T}$, et al. A randomised, double-blind, placebocontrolled trial of tropisetron in patients with schizophrenia. Ann Gen Psychiatry 2010;9:27.

12. Niitsu T, Fujisaki M, Shiina A, Yoshida T, Hasegawa T, Kanahara $\mathrm{N}$, et al. A randomized, double-blind, placebocontrolled trial of fluvoxamine in patients with schizophrenia: a preliminary study. J Clin Psychopharmacol 2012;32:593-601.

13. Niitsu T, Iyo M, Hashimoto K. Sigma-1 receptor agonists as therapeutic drugs for cognitive impairment in neuropsychiatric diseases. Curr Pharm Des 2012;18:875-883.

14. Huerta-Ramos E, Iniesta R, Ochoa S, Cobo J, Miquel E, Roca $\mathrm{M}$, et al. Effects of raloxifene on cognition in postmenopausal women with schizophrenia: a double-blind, randomized, placebo-controlled trial. Eur Neuropsychopharmacol 2014;24:223-231.

15. Lane HY, Lin CH, Green MF, Hellemann G, Huang CC, Chen PW, et al. Add-on treatment of benzoate for schizophrenia: a randomized, double-blind, placebo-controlled trial of D-amino acid oxidase inhibitor. JAMA Psychiatry 2013;70:1267-1275.

16. Keefe RS, Buchanan RW, Marder SR, Schooler NR, Dugar A, Zivkov M, et al. Clinical trials of potential cognitiveenhancing drugs in schizophrenia: what have we learned so far? Schizophr Bull 2013;39:417-435.

17. Do KQ, Conus P, Cuenod M. Redox dysregulation and oxidative stress in schizophrenia: nutrigenetics as a challenge in psychiatric disease prevention. World Rev Nutr Diet 2010;101:131-153.

18. Saruwatari J, Yasui-Furukori N, Kamihashi R, Yoshimori Y, Oniki K, Tsuchimine $\mathrm{S}$, et al. Possible associations between antioxidant enzyme polymorphisms and metabolic abnormalities in patients with schizophrenia. Neuropsychiatr Dis Treat 2013;9:1683-1698.

19. Yao JK, Keshavan MS. Antioxidants, redox signaling, and pathophysiology in schizophrenia: an integrative view. Anti- oxid Redox Signal 2011;15:2011-2035.

20. Bitanihirwe BK, Woo TU. Oxidative stress in schizophrenia: an integrated approach. Neurosci Biobehav Rev 2011;35:878-893.

21. Zhang XY, Chen da C, Xiu MH, Tang W, Zhang F, Liu L, et al. Plasma total antioxidant status and cognitive impairments in schizophrenia. Schizophr Res 2012;139:66-72.

22. Wu JQ, Chen da C, Tan YL, Tan SP, Wang ZR, Xiu MH, et al. Cognition impairment in schizophrenia patients with tardive dyskinesia: association with plasma superoxide dismutase activity. Schizophr Res 2014;152:210-216.

23. Zhang XY, Chen da C, Xiu MH, Tan YL, Yang FD, Zhang LY, et al. Clinical symptoms and cognitive impairment associated with male schizophrenia relate to plasma manganese superoxide dismutase activity: a case-control study. J Psychiatr Res 2013;47:1049-1053.

24. Gubert C, Stertz L, Pfaffenseller B, Panizzutti BS, Rezin GT, Massuda R, et al. Mitochondrial activity and oxidative stress markers in peripheral blood mononuclear cells of patients with bipolar disorder, schizophrenia, and healthy subjects. J Psychiatr Res 2013;47:1396-1402.

25. Reddy R, Reddy R. Antioxidant therapeutics for schizophrenia. Antioxid Redox Signal 2011;15:2047-2055.

26. Berk M, Copolov D, Dean O, Lu K, Jeavons S, Schapkaitz $\mathrm{I}$, et al. N-acetyl cysteine as a glutathione precursor for schizophrenia--a double-blind, randomized, placebo-controlled trial. Biol Psychiatry 2008;64:361-368.

27. Yao JK, Reddy RD, van Kammen DP. Oxidative damage and schizophrenia: an overview of the evidence and its therapeutic implications. CNS Drugs 2001;15:287-310.

28. Zhang XY, Zhou DF, Zhang PY, Wu GY, Su JM, Cao LY. A double-blind, placebo-controlled trial of extract of Ginkgo biloba added to haloperidol in treatment-resistant patients with schizophrenia. J Clin Psychiatry 2001;62:878-883.

29. Emsley R, Myburgh C, Oosthuizen P, van Rensburg SJ. Randomized, placebo-controlled study of ethyl-eicosapentaenoic acid as supplemental treatment in schizophrenia. Am J Psychiatry 2002;159:1596-1598.

30. Mahadik SP, Evans D, Lal H. Oxidative stress and role of antioxidant and omega-3 essential fatty acid supplementation in schizophrenia. Prog Neuropsychopharmacol Biol Psychiatry 2001;25:463-493.

31. Peet M, Brind J, Ramchand CN, Shah S, Vankar GK. Two double-blind placebo-controlled pilot studies of eicosapentaenoic acid in the treatment of schizophrenia. Schizophr Res 2001;49:243-251.

32. Peet M, Horrobin DF; E-E Multicentre Study Group. $A$ dose-ranging exploratory study of the effects of ethyleicosapentaenoate in patients with persistent schizophrenic symptoms. J Psychiatr Res 2002;36:7-18.

33. Fenton WS, Dickerson F, Boronow J, Hibbeln JR, Knable M. A placebo-controlled trial of omega-3 fatty acid (ethyl eicosapentaenoic acid) supplementation for residual symptoms and cognitive impairment in schizophrenia. Am J Psychiatry 2001;158:2071-2074.

34. Chengappa KN, Turkin SR, DeSanti S, Bowie CR, Brar JS, Schlicht PJ, et al. A preliminary, randomized, double-blind, placebo-controlled trial of L-carnosine to improve cognition in schizophrenia. Schizophr Res 2012;142:145-152.

35. Juge N, Mithen RF, Traka M. Molecular basis for chemoprevention by sulforaphane: a comprehensive review. Cell Mol Life Sci 2007;64:1105-1127.

36. Fahey JW, Haristoy X, Dolan PM, Kensler TW, Scholtus I, Stephenson KK, et al. Sulforaphane inhibits extracellular, intracellular, and antibiotic-resistant strains of Helicobacter 
pylori and prevents benzo[a]pyrene-induced stomach tumors. Proc Natl Acad Sci U S A 2002;99:7610-7615.

37. Chen $\mathrm{H}$, Wu J, Zhang J, Fujita Y, Ishima $\mathrm{T}$, Iyo $\mathrm{M}$, et al. Protective effects of the antioxidant sulforaphane on behavioral changes and neurotoxicity in mice after the administration of methamphetamine. Psychopharmacology (Berl) 2012;222:37-45.

38. Shirai Y, Fujita Y, Hashimoto K. Effects of the antioxidant sulforaphane on hyperlocomotion and prepulse inhibition deficits in mice after phencyclidine administration. Clin Psychopharmacol Neurosci 2012;10:94-98.

39. Hashimoto K. Brain-derived neurotrophic factor as a biomarker for mood disorders: an historical overview and future directions. Psychiatry Clin Neurosci 2010;64:341-357.

40. Hashimoto K. Sigma-1 receptor chaperone and brain-derived neurotrophic factor: emerging links between cardiovascular disease and depression. Prog Neurobiol 2013;100:15-29.

41. American Psychiatric Association. Diagnostic and statistical manual of mental disorders: DSM-IV-TR. 4th ed. Arlington, VA, USA: American Psychiatric Press; 2000.

42. Yoshida T, Suga M, Arima K, Muranaka Y, Tanaka T, Eguchi $\mathrm{S}$, et al. Criterion and construct validity of the CogState Schizophrenia Battery in Japanese patients with schizophrenia. PLoS One 2011;6:e20469.

43. Lim YY, Jaeger J, Harrington K, Ashwood T, Ellis KA, Stöffler A, et al. Three-month stability of the CogState brief battery in healthy older adults, mild cognitive impairment, and Alzheimer's disease: results from the Australian Imaging, Biomarkers, and Lifestyle-rate of change substudy (AIBL-ROCS). Arch Clin Neuropsychol 2013;28:320-330.

44. Kay SR, Fiszbein A, Opler LA. The positive and negative syndrome scale (PANSS) for schizophrenia. Schizophr Bull 1987;13:261-276.

45. Busner J, Targum SD. The clinical global impressions scale: applying a research tool in clinical practice. Psychiatry (Edgmont) 2007:4:28-37.

46. Kern RS, Nuechterlein KH, Green MF, Baade LE, Fenton WS, Gold JM, et al. The MATRICS Consensus Cognitive Battery, part 2: co-norming and standardization. Am J Psychiatry 2008;165:214-220.

47. Nuechterlein KH, Green MF, Kern RS, Baade LE, Barch $\mathrm{DM}$, Cohen JD, et al. The MATRICS Consensus Cognitive Battery, part 1: test selection, reliability, and validity. Am J Psychiatry 2008;165:203-213.

48. Pietrzak RH, Snyder PJ, Jackson CE, Olver J, Norman T, Piskulic D, et al. Stability of cognitive impairment in chronic schizophrenia over brief and intermediate re-test intervals. Hum Psychopharmacol 2009;24:113-121.

49. Maruff P, Thomas E, Cysique L, Brew B, Collie A, Snyder $\mathrm{P}$, et al. Validity of the CogState brief battery: relationship to standardized tests and sensitivity to cognitive impairment in mild traumatic brain injury, schizophrenia, and AIDS dementia complex. Arch Clin Neuropsychol 2009;24:165-178.

50. Yoshida T, Ishikawa M, Niitsu T, Nakazato M, Watanabe $\mathrm{H}$, Shiraishi $\mathrm{T}$, et al. Decreased serum levels of mature brain-derived neurotrophic factor (BDNF), but not its precursor proBDNF, in patients with major depressive disorder. PLoS One 2012;7:e42676.

51. Wild K, Howieson D, Webbe F, Seelye A, Kaye J. Status of computerized cognitive testing in aging: a systematic review. Alzheimers Dement 2008;4:428-437.

52. Fredrickson J, Maruff P, Woodward M, Moore L, Fredrickson A, Sach J, et al. Evaluation of the usability of a brief computerized cognitive screening test in older people for epidemiological studies. Neuroepidemiology 2010;34:65-75.

53. Ichimura S, Ohira T, Kobayashi M, Kano T, Akiyama T, Orii $\mathrm{M}$, et al. Assessment of cognitive function before and after surgery for posterior cranial fossa lesions using computerized and conventional tests. Neurol Med Chir (Tokyo) 2010;50:441-448.

54. Lim YY, Ellis KA, Harrington K, Ames D, Martins RN, Masters CL, et al. Use of the CogState Brief Battery in the assessment of Alzheimer's disease related cognitive impairment in the Australian Imaging, Biomarkers and Lifestyle (AIBL) study. J Clin Exp Neuropsychol 2012;34:345-358.

55. Mas S, Gassó P, Trias G, Bernardo M, Lafuente A. Sulforaphane protects SK-N-SH cells against antipsychoticinduced oxidative stress. Fundam Clin Pharmacol 2012;26: 712-721.

56. Falleti MG, Maruff P, Collie A, Darby DG. Practice effects associated with the repeated assessment of cognitive function using the CogState battery at 10-minute, one week and one month test-retest intervals. J Clin Exp Neuropsychol 2006;28:1095-1112.

57. Pietrzak RH, Olver J, Norman T, Piskulic D, Maruff P, Snyder PJ. A comparison of the CogState Schizophrenia Battery and the Measurement and Treatment Research to Improve Cognition in Schizophrenia (MATRICS) Battery in assessing cognitive impairment in chronic schizophrenia. $J$ Clin Exp Neuropsychol 2009;31:848-859.

58. Darby DG, Pietrzak RH, Fredrickson J, Woodward M, Moore L, Fredrickson A, et al. Intraindividual cognitive decline using a brief computerized cognitive screening test. Alzheimers Dement 2012;8:95-104.

59. Hammers D, Spurgeon E, Ryan K, Persad C, Heidebrink J, Barbas $\mathrm{N}$, et al. Reliability of repeated cognitive assessment of dementia using a brief computerized battery. Am J Alzheimers Dis Other Demen 2011;26:326-333.

60. Cole WR, Arrieux JP, Schwab K, Ivins BJ, Qashu FM, Lewis SC. Test-retest reliability of four computerized neurocognitive assessment tools in an active duty military population. Arch Clin Neuropsychol 2013;28:732-742.

61. Murthy NV, Mahncke H, Wexler BE, Maruff P, Inamdar A, Zucchetto $\mathrm{M}$, et al. Computerized cognitive remediation training for schizophrenia: an open label, multi-site, multinational methodology study. Schizophr Res 2012;139:87-91.

62. Green MJ, Matheson SL, Shepherd A, Weickert CS, Carr VJ. Brain-derived neurotrophic factor levels in schizophrenia: a systematic review with meta-analysis. Mol Psychiatry 2011;16:960-972.

63. Pandya CD, Kutiyanawalla A, Pillai A. BDNF-TrkB signaling and neuroprotection in schizophrenia. Asian $J$ Psychiatr 2013;6:22-28.

64. Carlino D, De Vanna M, Tongiorgi E. Is altered BDNF biosynthesis a general feature in patients with cognitive dysfunctions? Neuroscientist 2013;19:345-353.

65. Nurjono M, Lee J, Chong SA. A review of brain-derived neurotrophic factor as a candidate biomarker in schizophrenia. Clin Psychopharmacol Neurosci 2012;10:61-70. 